\title{
A Superbend X-Ray Microdiffraction Beamline at the Advanced Light Source
}

\author{
N. Tamura, M. Kunz, K. Chen, R.S. Celestre, A.A. MacDowell and T. Warwick \\ Lawrence Berkeley National Laboratory, 1 Cyclotron Road, Berkeley CA 94720 \\ Corresponding author: N. Tamura, email: ntamura@lbl.gov
}

\begin{abstract}
Beamline 12.3.2 at the Advanced Light Source is a newly commissioned beamline dedicated to $\mathrm{x}$-ray microdiffraction. It operates in both monochromatic and polychromatic radiation mode. The facility uses a superconducting bending magnet source to deliver an X-ray spectrum ranging from 5 to $22 \mathrm{keV}$. The beam is focused down to $\sim 1 \mathrm{um}$ size at the sample position using a pair of elliptically bent KirkpatrickBaez mirrors enclosed in a vacuum box. The sample placed on high precision stages can be raster-scanned under the microbeam while a diffraction pattern is taken at each step. The arrays of diffraction patterns are then analyzed to derive distribution maps of phases, strain/stress and/or plastic deformation inside the sample.
\end{abstract}

Keywords: $x$-ray microdiffraction, $x$-ray beamline, Laue diffraction, strain/stress measurements, microprobe

\section{Introduction}

Over the years, X-ray microdiffraction ( $\mathrm{UXRD}$ ) has become one of the several standard tools used at synchrotron facilities to identify and characterize materials at micron- and submicron scale. X-ray beam sizes and therefore spatial resolutions range from a few microns down to a few tens of nanometers depending on the source, photon energy range, focusing optics and other beamline characteristics. ${ }^{[1-3]} \mathrm{X}$-ray microdiffraction is often used in complement to microspectroscopy techniques such as microfluorescence (uXRF), micro X-ray absorption near-edge structure (uXANES) and micro- extended X-ray absorption fine structure ( uEXAFS) spectroscopies and is mainly a phase identification tool for minute or highly heterogeneous samples.

A typical modern microdiffraction setup delivers a focused monochromatic beam onto the sample and is equipped with an X-ray area detector mounted on a diffractometer to collect 2D diffraction patterns in transmission mode (requiring the use of a beam stop). Data are then analyzed via software such as Fit2D. ${ }^{[4]}$ A few beamlines at the Advanced Photon Source (APS), Advanced Light Source (ALS), European Synchrotron Radiation Facility (ESRF), National Synchrotron Light Source (NSLS), Canadian Light Source (CLS), Swiss Light Source (SLS) and Pohang Light Source (PLS) offer a more or less dedicated microdiffraction station with enhanced capabilities such as polychromatic beam for strain measurement and 3-dimensional (depth-resolving) X-ray diffraction. ${ }^{\text {[5-6] }}$ 
Along with groups at the APS and NSLS, the ALS has pioneered some of these techniques and has offered a dedicated poly/monochromatic station on the bending magnet beamline 7.3.3 open to the users community since 2001. ${ }^{[6]}$ The user program on ALS beamline 7.3.3 has proven to be highly successful. However, with the increasing complexity of new scientific problems a number of potential limitations in beamline capabilities became apparent. These limitations include limited spatial resolution (around $1 \mathrm{um}$ ) and strain resolution (typically $\left.2.10^{-4}\right)$, limited energy range (5-12 keV), limited flux in monochromatic mode, and time-consuming data collection and analysis. Exchanging a regular ALS bending magnet for a superconducting magnet is a good way to address most of these issues (Fig.1). For instance, the energy range can be extended and the increased flux at high energy allows using a smaller source size and thus obtaining a smaller focus spot size by flux trade-off. Increased flux also decreases data collection time and improves strain resolution through better statistics provided by an increased number of Laue spots. Higher energy photons also open the possibility of depth profiling techniques as the penetration depth in the sample increases.

This paper briefly describes the beamline optic components and goes over some of the improved capabilities and benefits obtained from the move of the ALS X-ray microdiffraction program to a superconducting bending magnet source. A concluding section will go over some of the first scientific applications that came out of that beamline.

\section{Beamline description}

The general design of the beamline is similar to the previous end-station on ALS beamline 7.3.3 ${ }^{[6]}$. Only a few parts of the original beamline have been recycled to the new one (tungsten rotary slits, X-ray CCD detector and diffractometer $2 \theta$ arm). Figure 2 shows the outline of the beamline in elevation and plan views. The beamline uses one of the three 6 Tesla superconducting magnet sources of the Advanced Light Source which provides a critical energy at about $12 \mathrm{keV}$, extending the photon spectrum range well into the hard x-ray regime. The first optic encountered by the x-ray beam is a horizontally deflecting toroidal mirror that conveys the beam to a virtual source just after the experimental hutch entrance. The $\mathrm{Pt}(25 \mathrm{~nm})$ and $\mathrm{Rh}(8 \mathrm{~nm})$ coated silicon toroidal mirror operates at a grazing angle of $3.5 \mathrm{mrad}$ cutting off the energy spectrum at about $22 \mathrm{keV}$. Water-cooled tungsten roll slits are used to adjust the virtual source size and therefore the size of the focused beam onto the sample. Final focusing is achieved using a KirkpatrickBaez (KB) mirror assembly contained in a vacuum box. The KB mirrors assembly is Peltier-cooled to compensate for the heat load on the mirrors inside the optic box vacuum chamber. The demagnifications to obtain a $1 \mathrm{x} 1 \mathrm{um}^{2}$ size beam are approximately 16:1 and 8:1 for the vertical and horizontal KBs respectively. The choice of using KBs is directed by the use of polychromatic radiation, as they are to date the most efficient achromatic optics available. The beamline can switch between white and monochromatic beam by way of a 2 channel-cut - 4 bounces $\mathrm{Si}(111)$ monochromator. This particular design is aimed for having the possibility to illuminate the same spot on the sample with either white or monochromatic beam independent of energy. The two channel-cuts are 
mounted on roll-bearing rotation stages that are independently driven by linear motors via a sine bar mechanism, replacing the previous 7.3.3 technology that used a tape-drive system. The sample stages are placed on a high precision XYZ stage for accurate positioning. A $\chi$ stage defines the incident beam angle onto the sample. Diffraction patterns are collected using a MAR133 X-ray detector and X-ray fluorescence signal by a Vortex Si-drift detector coupled with a XIA multi channel analyzer. The design is flexible to allow for both reflective and transmitting sample geometries. Precise positioning (within a few microns) of the sample into the focal point of the beam is obtained via a Keyence laser triangulation tool.

The main differences with the previous bending magnet beamline are the extended energy range (up to $22 \mathrm{keV}$ ) and increased flux allowing scaling down the exposure time for diffraction patterns by almost an order of magnitude. Using polychromatic radiation, strain states in the sample are obtained by fitting the angular differences between reflections using a non-linear least square method. Strain resolution depends on the number of reflections used in the fit. By extending the available energy range from 12 $\mathrm{keV}$ to $22 \mathrm{keV}$, the number of available spots significantly increases, allowing for almost an order of magnitude better strain resolution if compared to identical material as measured on ALS beamline 7.3.3 (from $210^{-4}$ down to $510^{-5}$ for a typical metal sample) (Figure 3). Another difference with the previous system is the use of a vacuum box instead of a He-filled box for the optics, giving rise to additional flux gain although the main reason is more practical as no gas flow is necessary and risk of ozone formation is minimized. Apart from the M1 mirror, all the optical components of the beamline are inside a vacuum chamber sitting on an optical table inside the experimental hutch, and evacuated first by a dry roughing pump and then by a turbo pump that provides a vacuum of about $10^{-5}$ Torr. Some extra care has been taken to enhance the mechanical coupling between the optic box and the sample stages to limit differential vibrations as well as temperature drifts. The hutch itself is maintained at a constant temperature of $22+/-0.1$ ${ }^{\circ} \mathrm{C}$. by an air conditioning system. The tungsten rotary slits are water-cooled, while the monochromator crystals and the KB assembly use a water-based Peltier cooling system.

\section{Beamline performance and capabilities}

Beam spot size has been minimized using an on-line KB adjustment system. This consists of a pair of adjustable size slits acting as a pinhole upstream of the KBs and a scintillator $\left(1 \mathrm{~mm}\right.$ thick piece of single crystal $\mathrm{CdWO}_{4}$ ) and an optical CCD camera equipped with a zoom lens. The system is positioned at the x-ray beam focal point. The technique uses the Hartmann method to adjust the bends and pitch of the KB mirrors. ${ }^{[7]}$ With this technique, mirror figure errors of 0.2 urad can be measured and corrected for. The size of the spot has been measured by a knife-edge method using a $150 \mathrm{um}$ diameter tungsten wire. The best focus obtained with the current KB mirrors assembly was $630 \mathrm{~nm}$ (h) x $500 \mathrm{~nm}$ (v) (Fig. 4). This focus however has slightly degraded over time but stays stable around $1 \mathrm{um}$ for several months. Vibrations of the sample stage relative to the optics box have been measured at $\sim 500 \mathrm{~nm} \mathrm{rms}$ and are probably one of the main factor preventing us from obtaining a sub $500 \mathrm{~nm}$ spot size, although it is not the limiting factor for the current 1 
um size beam. The vibration problem is in the process of being remediated by a clamping system that will reduce the mechanical loop between the optic box and the upper sample stages while the $\chi$ motor is unused.

Key to the success of the microdiffraction program is the ability to extract quantitative information from both white and monochromatic beam patterns. On beamline 12.3.2, this is achieved by using an in-house continuously evolving dedicated software called XMAS (X-ray Microdiffraction Analysis Software). ${ }^{[6]}$ This software's main feature is its ability to index thousands of patterns in an automated way and convert the information extracted from individual patterns (such as crystal orientation and strain) into two-dimensional maps. The indexing algorithm consists in finding angular matches of 3 non-collinear experimental scattering vectors calculated from the spot position on the X-ray CCD detector with those calculated from a known structure. This simple algorithm proved to be highly robust allowing indexing up to 100 grains in a single "polycrystal" Laue pattern or analyzing Laue patterns with fuzzy and/or highly distorted peaks due to mechanical deformations. It tends however to be time-consuming in the cases of low symmetry structures (triclinic, monoclinic) and large unit cell phases, but works well for most applications in metal physics.

\section{Applications}

The beamline uses two modes of operations that can be employed in a complementary way (Fig 5). For both modes, the sample can be raster-scanned under the X-ray microbeam while X-ray diffraction patterns or a fluorescence signal are collected at each step. No sample rotation is required. This avoids problems related to sphere of confusion or non-constant diffraction volume. In polychromatic mode, polycrystalline samples consisting of grains with sizes larger than the beam size (1 micron and more) as well as smaller isolated single crystalline particles (such as single nanowires) can be investigated. Each Laue pattern comes from a single crystal or a limited number of single crystals and is readily indexed using the XMAS code. Besides grain orientation, Laue patterns can also be used to measure the deviatoric strain as well as to identify and measure the distribution of geometrically necessary dislocations (Fig 6). ${ }^{[8]}$

One recent example using polychromatic microdiffraction is the study of the ordering of nacre in a red abalone shell. ${ }^{[9]}$ Nacre is a composite biomaterial made of $95 \%$ aragonite and 5\% various proteins, essentially chitin. It is 3000 times tougher than aragonite, a feat that is far from being achieved by any man-made composites. In order to understand the mechanism of nacre formation, polychromatic X-ray microdiffraction has been used to complement X-PEEM (X-Ray photoelectron emission microspectroscopy) imaging results. Polychromatic X-ray microdiffraction indicates that the material is made up of aragonite tablets with a c-axis spread that starts from approximately $24^{\circ}$ at the prismatic calcite-nacre interface and goes down to $6^{\circ}$ a few tens of microns away (Fig. 7). This gradual ordering is compatible with a competitive growth mechanism where the crystallites with a c-axis normal to the interface are kinetically favored. 
A second recent application of polychromatic X-ray microdiffraction is the study of the electromigration effect in a $\mathrm{Sn}-\mathrm{Cu}$ solder joint as they are used in lead-free flip-chip technology. ${ }^{[10]}$ Grain orientation has been carefully monitored over a time period of over 40 hours during an accelerated electromigration test with a current density of $1.2510^{4}$ $\mathrm{A} / \mathrm{cm}^{2}$ at $75^{\circ} \mathrm{C}$. It was observed that grains which are sitting in a current crowding region at the anode corner of the solder ball show significant rotation of up to $0.4^{\circ}$ over the length of the experiment, while no other grain in the sample displays such changes in orientation. Taking into account the anisotropy of the electrical conductivity in tin, it is found that these observed grain rotations are driven by a tendency to decrease the electrical resistivity in the solder bump.

Monochromatic beam mode X-ray microdiffraction is suitable for polycrystalline samples with grain size much smaller than the beam size (nanometric sizes). In that case, the diffraction patterns are powder patterns (Debye-Scherrer rings) for which the ring positions can be fitted against a crystal structure database allowing for phase identification. Slight distortions of the diffraction rings also provide information on the macrostrain while broadening is associated to particle sizes and/or microstrain.

Monochromatic beam X-ray microdiffraction finds most of its applications in phase identification in heterogeneous samples typical of environmental and geosciences. On beamline 12.3.2, it has been used for instance to identify components in the bright colored coatings (slips) of gallo-roman potteries dating from first century BC to the $2^{\text {nd }}$ century AD. ${ }^{[11]}$ One particular example of these tablewares is Terra Sigillata of the marbled type, where the slip is brightly colored in a mixture of red and yellow colors. Xray microfluorescence and monochromatic X-ray microdiffraction have been combined to identify the composition of the slip which consists of two layers, a 10 micron thick layer of red slip and a 30 microns thick layer of yellow slip sequentially dip-deposited at the surface of the pottery. ${ }^{[12]}$ The red slip turns out to be the standard hematite-based slip used in the conventional red Terra Sigillata, while the yellow color of the second slip was found to be due to a titanium-rich mineral called pseudobrookite $\left(\mathrm{Fe}_{2} \mathrm{TiO}_{5}\right)$. This yellow color can only be obtained through specific firing conditions indicative of the high level of mastery achieved by the ancient potters. Other phases that have been identified in the slips are a spinel $\left(\mathrm{MgAl}_{2} \mathrm{O}_{4}\right)$ in the yellow slip and corundum $\left(\mathrm{Al}_{2} \mathrm{O}_{3}\right)$ in both layers, while hematite and anorthite are ingredients of the body. Micrometric sized phases such as quartz have also been identified in a complementary way, using the polychromatic beam mode.

\section{Conclusions and future prospects}

Beamline 12.3.2 is a versatile X-ray microdiffraction station recently built at the Advanced Light Source on a superconducting magnet source. The beamline is fully operational and is now open to the user community. Some of the enhanced capabilities of the beamline compared to the old bending magnet beamline 7.3.3 are a higher photon flux on the sample, better strain resolution, broader energy bandpass, enhanced computing capabilities (use of 24 node dual processor Linux cluster) and enhanced sample positioning system. Problems currently or about to be addressed are: sub-500 nm 
spot size on the sample, sample stage vibrations issues, and reduced data collection time due to long detector readout time. Future work includes the full implementation of 3D Xray microdiffraction for depth-resolved studies and energy resolved Laue

microdiffraction for structure refinement of small crystals. The beamline is presently treating a broad range of applications including strain/stress measurements in microelectronic devices, and nanofabricated specimens, characterization of environmental and geological samples and general material science studies.

\section{Acknowledgements:}

The Advanced Light Source is supported by the Director, Office of Science, Office of Basic Energy Sciences, Materials Sciences Division, of the U.S. Department of Energy under Contract No. DE-AC02-05CH11231 at Lawrence Berkeley National Laboratory and University of California, Berkeley, California. The move of the micro-diffraction program from ALS beamline 7.3.3 onto the ALS superbend source 12.3.2 was enabled through the NSF grant \# 0416243 and Iowa State University. Special thanks to Matthew M. Church, Sirine Fakra, Edward E. Domning, James M. Glossinger, Brian V. Smith, Valeryi Yaschuck and Greg Morrison for their active participation to the commissioning of the beamline.

\section{References:}

[1] H. Mimura, H. Yumoto, S. Matsuyama, Y. Sano, K. Yamamura, Y. Mori, M. Yabashi, Y. Nishino, K. Tamasaku, T. Ishikawa, and K. Yamauchi, Efficient focusing of hard x rays to $25 \mathrm{~nm}$ by a total reflection mirror, Appl. Phys. Lett. 90(5) (2007) 059103.

[2] O. Hignette, P. Cloetens, G. Rostaing, P. Bernard, and C. Morawe, Efficient sub $100 \mathrm{~nm}$ focusing of hard x rays , Rev. Sci. Instrum. 76(6) (2005) 063709.

[3] W. J. Liu, G. E. Ice, J. Z. Tischler, A. Khounsary, C. Liu, L. Assoufid, and A. T. Macrander, Short focal length Kirkpatrick-Baez mirrors for a hard x-ray nanoprobe , Rev. Sci. Instrum. 76(11) (2005) 113701.

[4] A P Hammersley, FIT2D: An Introduction and Overview, ESRF Internal Report, ESRF97HA02T, (1997)

[5] B. C. Larson, W. Yang, G. E. Ice, J. D. Budai, and J. Z. Tischler, Three-dimensional X-ray structural microscopy with submicrometre resolution, Nature 415 (200), 887 .

[6] N. Tamura, A.A. MacDowell, R. Spolenak, B.C. Valek, J. C. Bravman, W.L. Brown, R. S. Celestre, H.A. Padmore, B.W. Batterman and J.R. Patel, Scanning X-ray Microdiffraction with submicron white beam for strain/stress and orientation mapping in thin films , J. Synchrotron Radiat. 10(2) (2003) 137-143. 
[7] J. Hartmann J, Bemerkungen uber den Bau und die Justirung von Spektrographen, Z. Instrumentenkunde, 20, 47 (1900).

[8] A.S. Budiman, W.D. Nix, N. Tamura, B.C. Valek, K. Gadre, J. Maiz, R. Spolenak, and J.R. Patel, Appl. Phys. Lett. 88, 233515 (2006).

[9] P. Gilbert, R. A. Metzler, D. Zhou, A. Scholl, A. Doran, A. Young, M. Kunz, N. Tamura, S. N. Coppersmith, Gradual Ordering in Red Abalone Nacre. J. Am. Chem. Soc. 130 (2008) 17519-17527

[10] K. Chen, N. Tamura, and K.-N. Tu, In-situ study of electromigration-induced orientation evolution in $\mathrm{Pb}$-free solder joint by synchrotron microdiffraction, MRS Proceedings (2008) in press.

[11] P. Sciau, P. Goudeau, N. Tamura and E. Dooryhee , Micro scanning X-ray diffraction study of Gallo Roman Terra Sigillata ceramics, Applied Physics A-Materials Science \& Processing 83 (2) (2006) 219224.

[12] Y. Leon, Ph. Sciau, Ph. Goudeau, N. Tamura, S. Webb, A. Mehta, The nature of marbled Terra Sigillata Slips: A combined $\mu \mathrm{XRF}$ and $\mu$ XRD investigations, J. Phys A. (2008) in press.

\section{Figure captions:}

Figure 1.- Comparison between the calculated flux at the sample position from a ALS regular 1.3 tesla bending magnet and from a 6 tesla superconducting magnet.

Figure 2.- Elevation and plan view of the 12.3.2 microdiffraction beamline at the Advanced Light Source.

Figure 3.- Strain value in function of number of Laue spots taken into account in the refinement for a nominally unstrained material. The error spread is diminishing as the number of Laue spot is increased.

Figure 4.- X-ray beam size measurements obtained by knife-edge on BL 12.3.2. The FWHM of the horizontal and vertical focus spots are $630 \mathrm{~nm}$ and $500 \mathrm{~nm}$, respectively.

Figure 5.- Polychromatic (white) beam is used for polycrystalline sample with grain size larger than the beam size, while monochromatic beam is used for polycrystalline sample with grain size smaller than the beam size.

Figure 6.- Example of outputs obtained from a polychromatic X-ray microdiffraction scan. The sample is an annealed Fe-47at\% $\mathrm{Cr}$ alloy consisting in a mixture of the BCC $\alpha$ phase (appearing in dark gray in the SEM image) and of a tetragonal $\sigma$ ' phase (appearing 
in white in the SEM image). The analysis of the Laue diffraction patterns yield the grain orientation maps of both phases as well as deviatoric strain and dislocation density maps.

Figure 7.- Spread of the aragonite c-axis angle with respect to the calcite/nacre interface normal in function of the distance from this interface. 
Figure 1

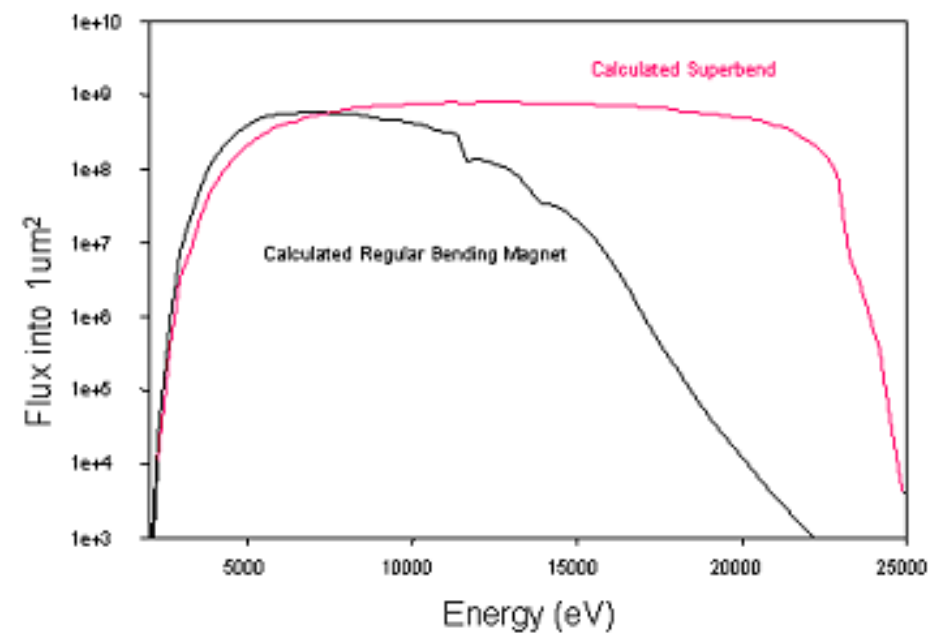


Figure 2

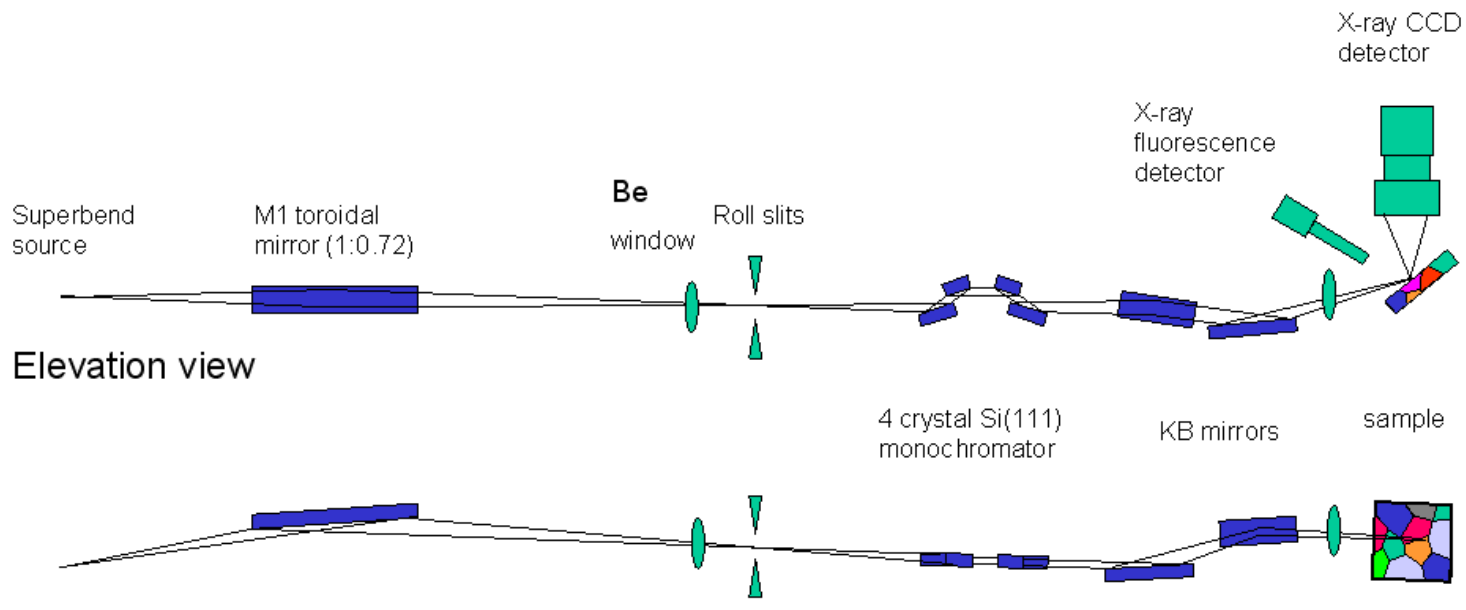

Plan View

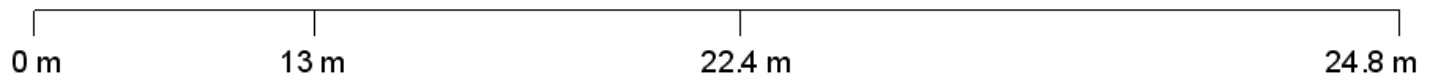


Figure 3

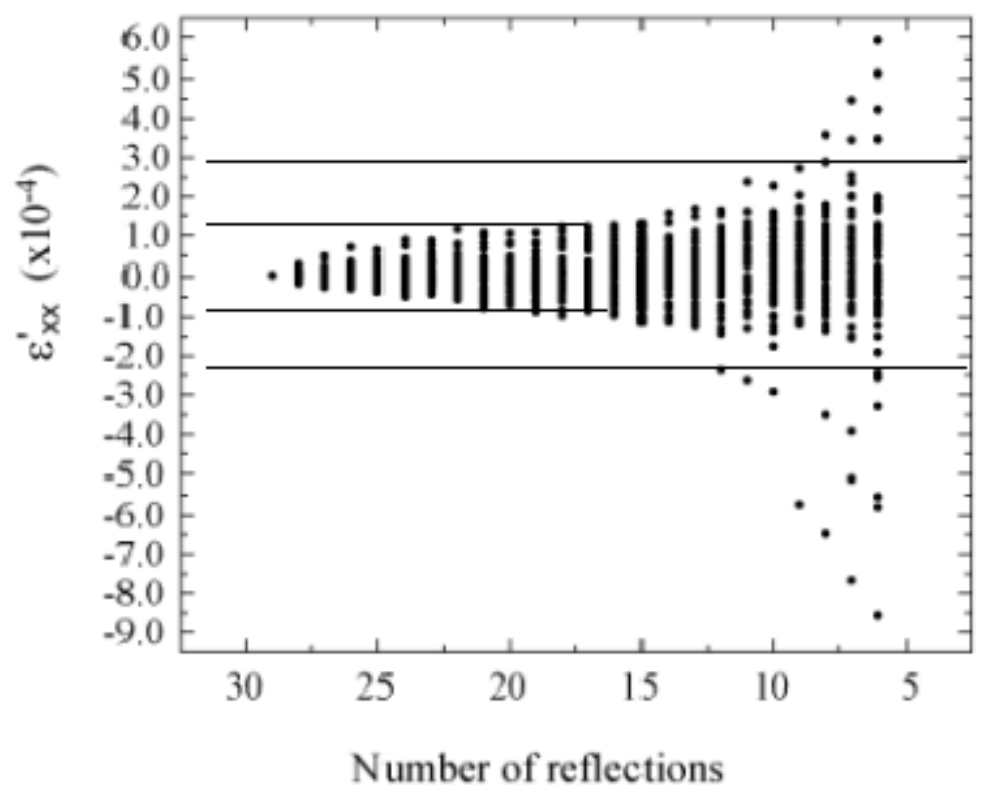


Figure 4

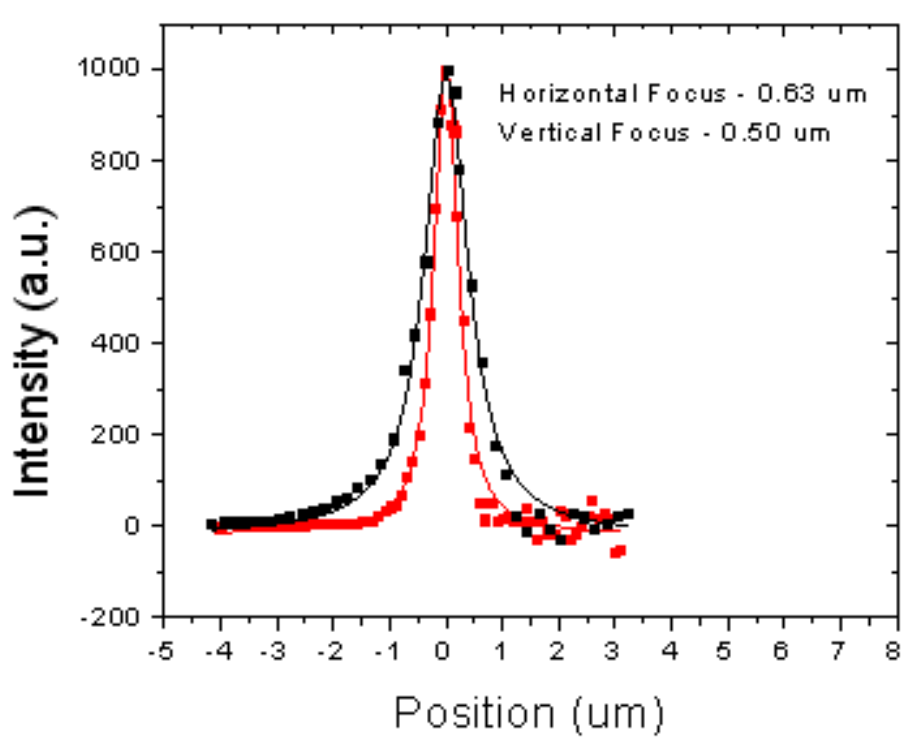


Figure 5

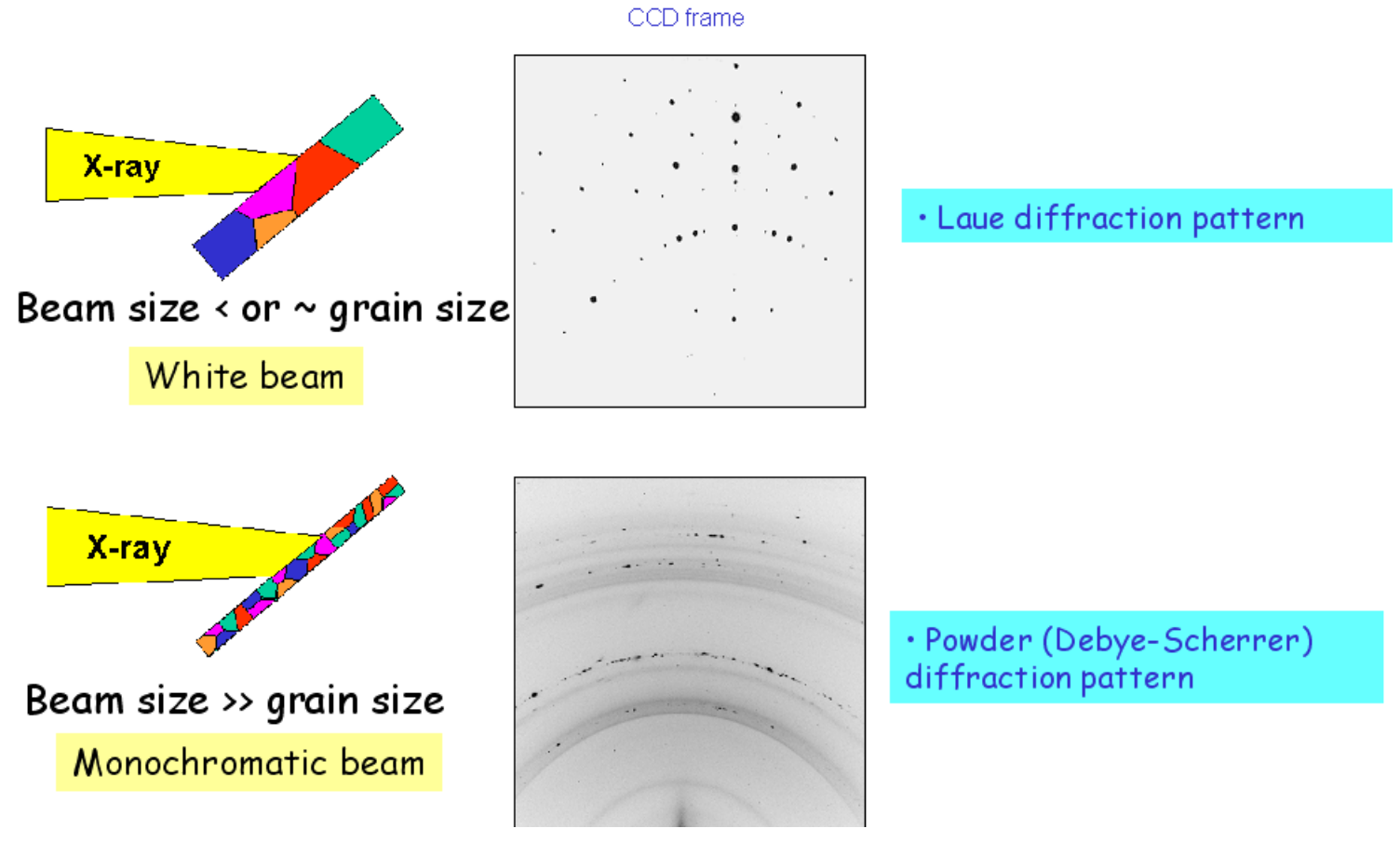


Figure 6

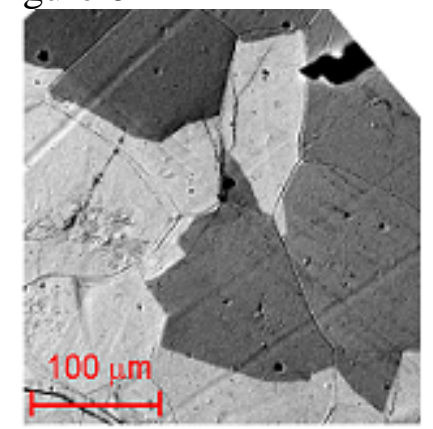

\section{Fe-Cr alloy}

$\alpha$-phase (BCC)

$\sigma$-phase (tetragonal)

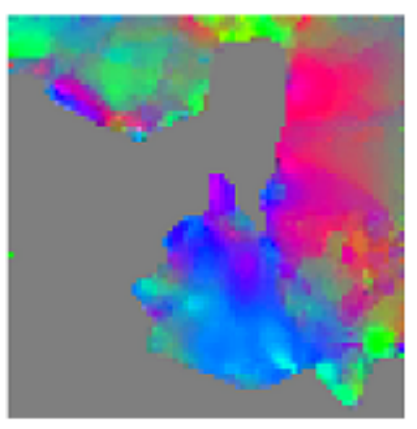

strain
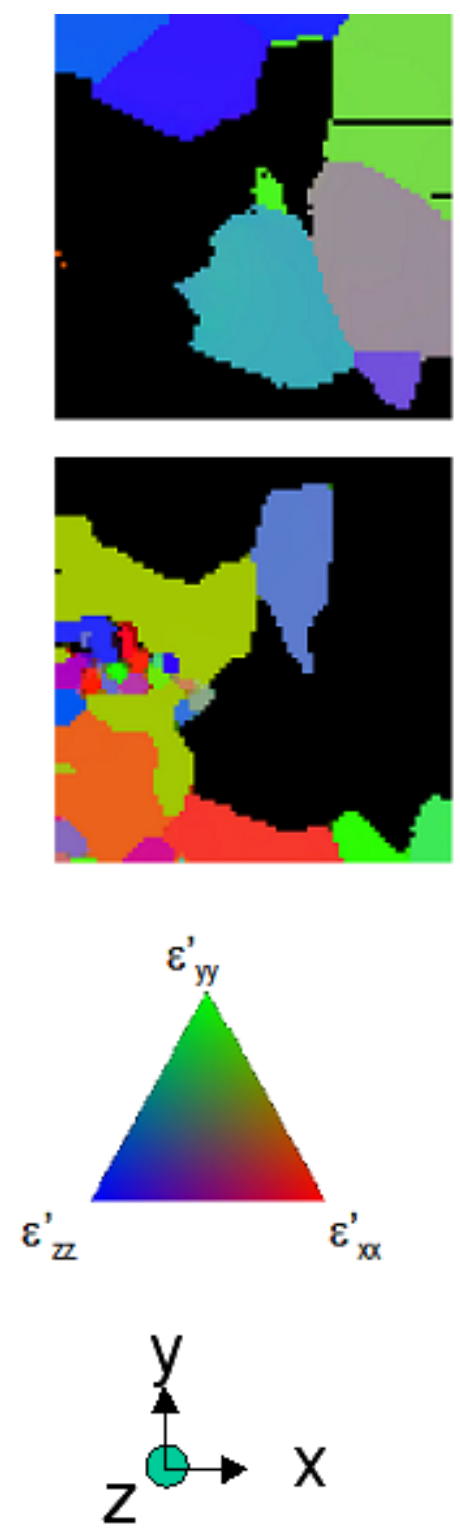

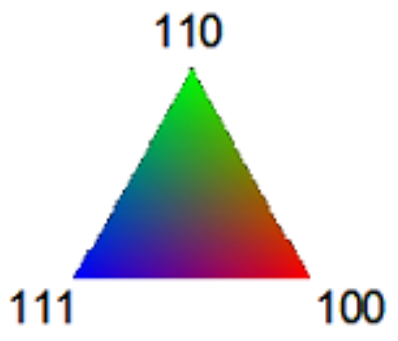

Orientation
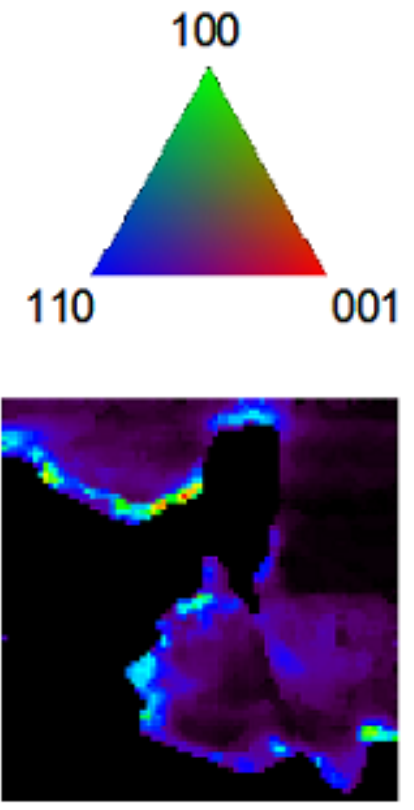

Dislocation density 
Figure 7

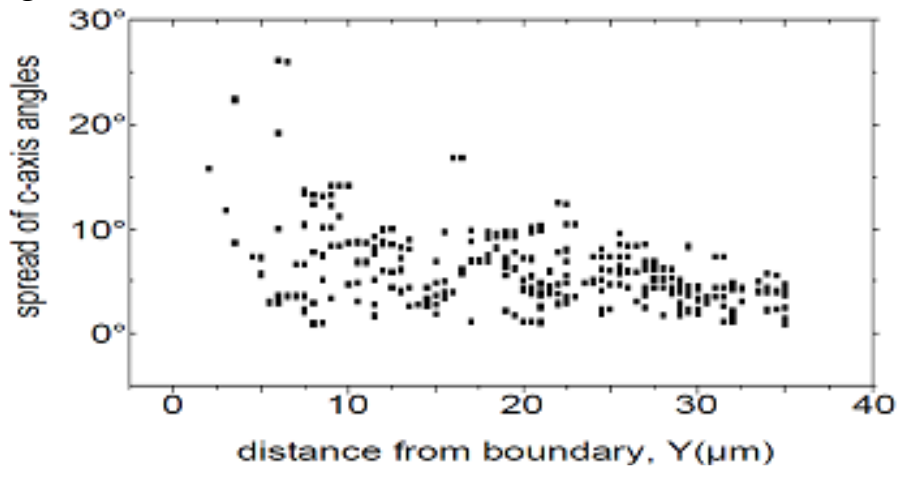

